\title{
CANCER
}

\section{Epidemiological differences between adenocarcinoma of the oesophagus and adenocarcinoma of the gastric cardia in the USA}

\author{
H B El-Serag, A C Mason, N Petersen, C R Key
}

See end of article for authors' affiliations

Correspondence to: Dr H B El-Serag, Houston VA Medical Center (152) 2002 Holcombe Boulevard, Houston, TX 77030, USA.

hasheme@bcm.tmc.edu

Accepted for publication 12 April 2001

\begin{abstract}
Background and aims: It has been suggested that gastric cardia adenocarcinoma (GCA) is a distinct entity from oesophageal adenocarcinoma (OA). We examined several epidemiological features of GCA and OA in the USA to elucidate differences/similarities between these malignancies.

Methods: Using the database of Surveillance, Epidemiology, and End Results (SEER) program, we examined incidence rates for temporal changes, and ethnic and age distributions, and performed birth cohort analyses for cases with morphologically and histologically confirmed OA or GCA.

Results: The age adjusted incidence rates of OA rose progressively, reaching 1.8 per $100000195 \%$ confidence interval 1.7-1.9) during 1987-1991 and 2.5 per 100000 (2.3-2.6) during 1992-1996. In 1992-1996, Whites were affected five times more than Blacks, and men eight times more than women. A significant increase in incidence occurred among younger persons aged 45-65 years. Irrespective of age, OA was characterised by higher incidence rates among more recent birth cohorts: a $40 \%$ increase in incidence for each five year increase in the date of birth - a "birth cohort effect". On the other hand, the incidence rates of GCA reached their highest level of 3.3 per 100000 (3.2-3.4) in 1987-1991 and subsequently declined during 1992-1996 to 3.1 per 100000 (3.0-3.3). Whites were affected twice more than blacks and men five times more than women. Most patients with GCA were older than 60 years with no increase among younger persons and no birth cohort effect $(p=0.99)$. Conclusion: Several significant epidemiological differences exist between OA and GCA. These differences suggest that these two malignancies are separate entities with different risk factors.
\end{abstract}

O esophageal adenocarcinoma (OA) has been reported as the fastest rising malignancy among White men in the USA. ${ }^{1-3}$ Patients with gastro-oesophageal reflux disease (GORD) are at an increased risk of developing intestinal metaplasia (IM) at the lower end of the tubular oesophagus (Barrett's oesophagus), which is the precursor lesion for OA. ${ }^{4}$ On the other hand, the incidence of adenocarcinoma of the stomach distal to the gastric cardia is progressively declining in developed countries, including the USA. ${ }^{1-3}$ Helicobacter pylori infection increases the risk of developing IM, atrophy, and adenocarcinoma of the stomach distal to the gastric cardia. ${ }^{5}$ The progressive reduction in the number of patients infected with $H$ pylori is thought to underlie the decline in cancer of the stomach distal to the gastric cardia. ${ }^{35}$

In between the lower end of the oesophagus and the distal stomach lies the gastric cardia. It remains unclear if IM arising in the gastric cardia is a distinct entity from that arising in the oesophagus. The incidence of gastric cardia adenocarcinoma (GCA) in the USA has increased but to a lesser extent than OA during the period between 1973 and the late 1980s. ${ }^{12}$ However, recent studies have reported the presence of significant epidemiological differences between the precursor lesions - that is, IM, of the oesophagus, and that of the gastric cardia. $^{6-9}$ Patients with Barrett's metaplasia were likely to be White men with evidence of GORD while patients with IM of the cardia were likely to be non-White with evidence of gastritis and $H$ pylori infection. ${ }^{6-9}$ It remains unclear however if such differences exist between the epidemiology and risk factors of OA and that of GCA. Recent studies by Lagergren et al have suggested that when these tumours were classified with a rigorous approach, there were clear differences in the risk factor profiles. ${ }^{10}{ }^{11}$ For example, GORD and obesity have been found to be strong independent risk factors for GCA in studies examining patients with GCA grouped with OA as "cardio- oesophageal" adenocarcinoma. ${ }^{12}{ }^{13}$ However, when these two malignancies are examined separately, the risks associated with GORD and/or obesity are much less strong for GCA than they are for OA. ${ }^{10} 11$

Examining the recent epidemiological trends of $\mathrm{OA}$ and GCA may help in elucidating differences/similarities between these malignancies. For example, if these two malignancies shared the same risk factor(s), one would expect similarities in the temporal trends of their incidence rates and distribution of affected persons by age, ethnicity, and birth cohort.

In the USA, the Surveillance, Epidemiology, and End Results (SEER) Program is an ongoing contract supported program of the National Cancer Institute to collect population based cancer incidence and survival data in a uniform cancer registry. All cancers in the SEER database are categorised based on their morphological as well as histological appearance. Data from the SEER database were previously used by Blot et al to report the incidence of OA and GCA through to 1994. ${ }^{12}$ In the current study, we use more up to date data from the SEER database to perform a birth cohort analysis and to compare the ethnic differences and changes in age distribution of these malignancies (OA and GCA) separately over the 20 year period between 1977 and 1996.

\section{METHODS}

Database

The study population was selected from the nine population based cancer registries that constitute the National Cancer

Abbreviations: OA, oesophageal adenocarcinoma; GCA, gastric cardia adenocarcinoma; GORD, gastro-oesophageal reflux disease; SEER, Surveillance, Epidemiology, and End Results; BMI, body mass index; IM, intestinal metaplasia. 


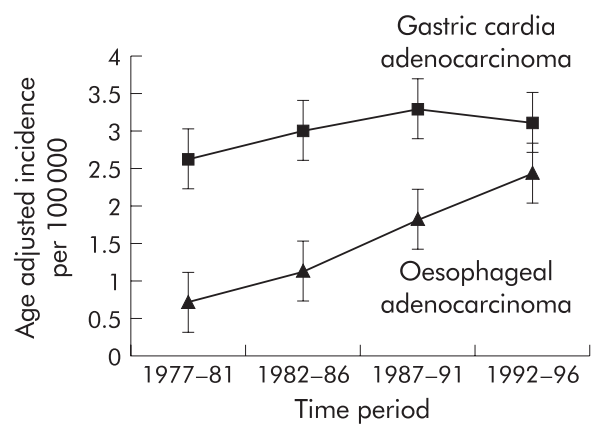

Figure 1 Overall age adjusted rates of oesophageal adenocarcinoma and gastric cardia adenocarcinoma per 100000 US population. The incidence rates are shown for five year periods between 1977 and 1996.

Institute's SEER Program. These registries account for 10-14\% of the US population and include the states of Connecticut, Hawaii, New Mexico, and Utah and four metropolitan areas of San Francisco/Oakland, Detroit, Seattle, and Atlanta. ${ }^{14}$ The SEER Program collects information on demographic characteristics of patients, anatomical site of malignancy, and histological cell type. The International Classification of Diseases for Oncology (ICD-O) is employed to encode for malignancies in the SEER incidence data. ${ }^{15}$

\section{Study population}

All patients who were diagnosed between 1977 and 1996 with a histologically confirmed OA or GCA were included. We used the site and histological type categories that are based on the ICD-O codes: oesophagus (C15-C15.9), gastric cardia (C16.0), and adenocarcinoma (8140-8573). ${ }^{15}$ Oesophagogastric junction tumours are included with gastric cardia under C16.0.

\section{Statistical analyses}

Cohorts of patients with either adenocarcinoma of the oesophagus or gastric cardia were analysed separately, based on the five year interval of cancer diagnosis: 1977-1981, 19821986, 1987-1991, and 1992-1996. Age adjusted incidence rates and their $95 \%$ confidence intervals (CIs) were calculated for each entire five year cohort as well as for subgroups based on sex (men versus women), and ethnicity (White, Black, other). The latter "other" category include Pacific Islanders, Asians, and Filipinos. The absence of a proper population denominator prohibited obtaining incidence rates on individual subgroups within this heterogeneous group. The US population in 1970 was used as the standard population for indirect age standardisation. All incidence rates were calculated using SEER*Stat version 2.0, which is the statistical package created for analysis of the SEER database. ${ }^{14}$ Incidence rates were considered significantly different if their 95\% CI did not overlap.

To examine the age distribution of cancer cases, the age specific incidence rates for each malignancy were plotted separately for each of the consecutive five year periods between 1977 and 1996.

Subsequently, the age specific incidence rates of all subjects diagnosed in SEER with either OA or GCA between 1977 and 1996 were analysed against their period of birth. Approximate birth cohorts were established for cases diagnosed between 1977 and 1996. It was assumed that subjects in the age group 60-65 years during 1982-1986, those in the age group 65-69 years during 1987-91, and those in the age group 70-75 years during 1991-1996 were born in the period centred on 1924. As the age of these patients at the time of cancer diagnosis ranged between 20 and 85 years, their dates of birth varied between 1894 and 1974.

A birth cohort is defined as a group of subjects born during the same time period. A cohort effect is the variation in a
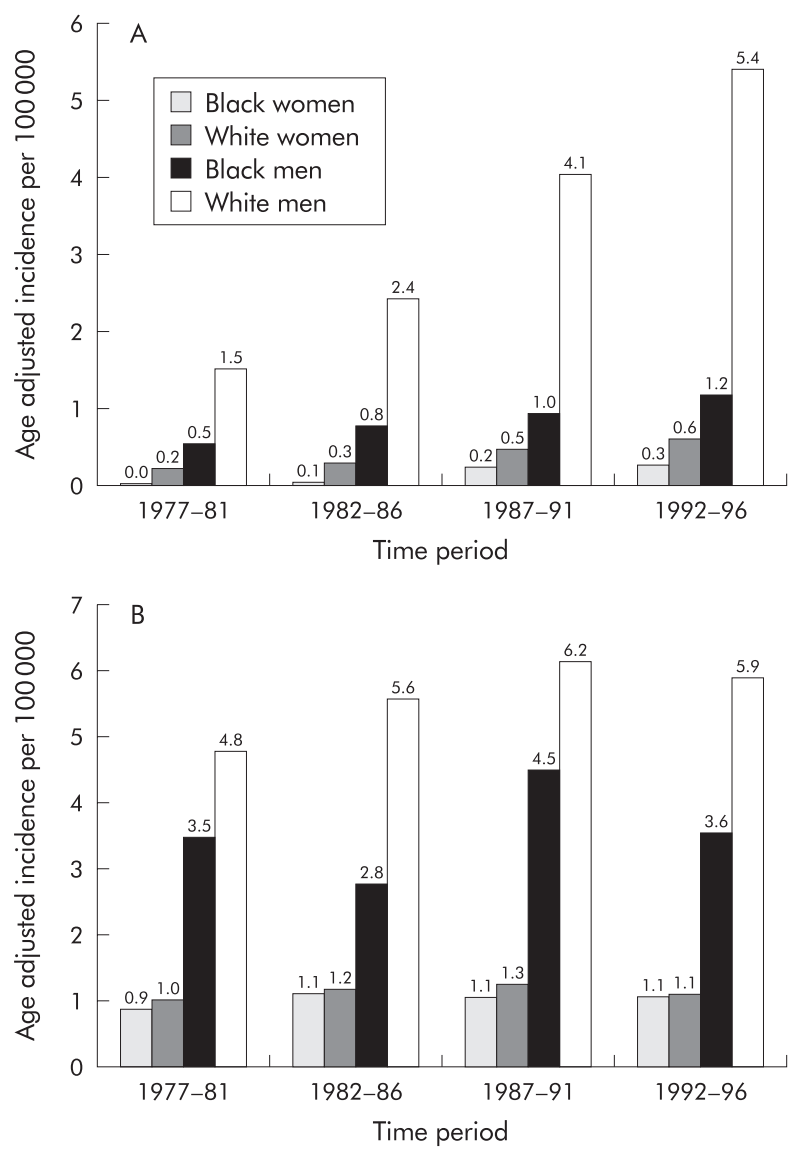

Figure 2 Incidence of oesophageal adenocarcinoma (A) and gastric cardia adenocarcinoma $(B)$ in different sex and ethnic groups. For each of the five year periods between 1977 and 1996, the age adjusted rates are shown for Black women, White women, Black men, and White men.

health status measure, such as the incidence of disease, that results from different exposures of the birth cohorts to risk factors that may change over time ${ }^{16}{ }^{17} \mathrm{~A}$ birth cohort analysis such as that of Banatavla et al was used to examine the cohort effect. ${ }^{16}$ A logistic regression model, using group level data, was run separately for the two diseases. Each analysis was based on 56 observations representing groups formed using the year of birth of the group and their age in one of the four time periods: 1977-1981, 1982-1986, 1987-1991, and 19921996. The year of birth was categorised into 17 groups ranging from the five years centred around 1894 to the five years centred around 1974. Age was categorised into 13 groups consisting of 12 five year groups ranging from ages 20-24 to ages 80-84, and one group aged 85 and older. For each age group, there were four birth cohorts representing patients who were the same age in the time period 1977-1996. For example, those aged 25-29 years in 1977-1996 were members of the 1954, 1959, 1964, and 1969 birth cohorts.

The events/trial syntax of the logistic regression procedure in SAS was used for analysis. Outcome was modelled as the number of cases of disease diagnosed in the group out of the total US population of equal age during the time interval. Independent variables included terms for the cohort, age at diagnosis, and a cohort-age interaction. Variables were considered statistically significant if the $\mathrm{p}$ value for the Wald $\chi^{2}$ statistic was 0.05 or less.

\section{RESULTS}

During the time period 1977-1996, 4751 patients were identified with morphologically and histologically proved OA and 9157 patients with GCA in the SEER database. 

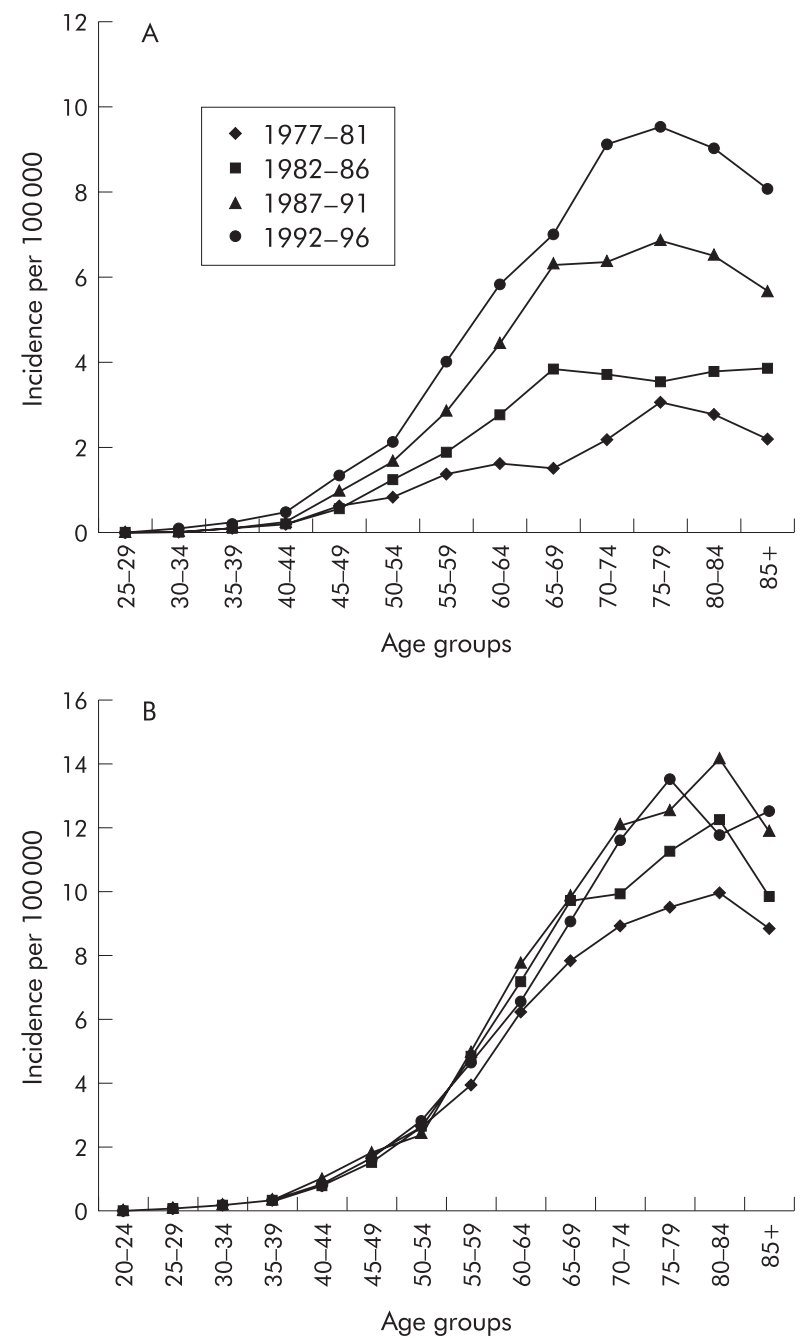

Figure 3 Age distribution of cases diagnosed with oesophageal adenocarcinoma (A) and gastric cardia adenocarcinoma $(B)$ in the USA between 1977 and 1996. Each of the individual curves represents the age specific incidence rates in a five year period.

The overall age standardised incidence rates for each malignancy are shown in fig 1 . The age adjusted incidence rate for OA progressively increased from 1977, reaching 1.8 per 100000 (95\% CI 1.7-1.9) during 1987-1991 and 2.5 per 100000 (95\% CI 2.3-2.6) during 1992-1996. On the other hand, adenocarcinoma of the gastric cardia reached its highest age adjusted incidence rate of 3.3 per 100000 (95\% CI 3.2-3.4) during 1987-1991 and then subsequently levelled off to 3.1 per 100000 (95\% CI 3.0-3.3) during 1992-1996.

Figure $2 \mathrm{~A}$ illustrates the fact that the age adjusted incidence rates of OA were 6-8 times higher in men than in women and 3-4 times higher in Whites than in Blacks. The progressive increase in incidence affected Whites and Blacks of both sexes. However, White men constituted $82 \%$ of all cases with OA and they experienced most of the witnessed rise in incidence rates. In 1992-1996, White men were affected 4.5 times more than Black men.

Compared with OA, the discrepancy between ethnic groups and sex was less pronounced in GCA (fig 2B). Men were affected 3-5 times more than women, and Whites were affected 1.5-2 times more with GCA than Blacks. In 1992-1996, White men were affected 1.7 times more than Black men.

In fig $3 \mathrm{~A}$, the age distribution of all incident cases of $\mathrm{OA}$ is shown for consecutive five year time intervals between 1977 and 1996. The age specific incidence increased until 75-79
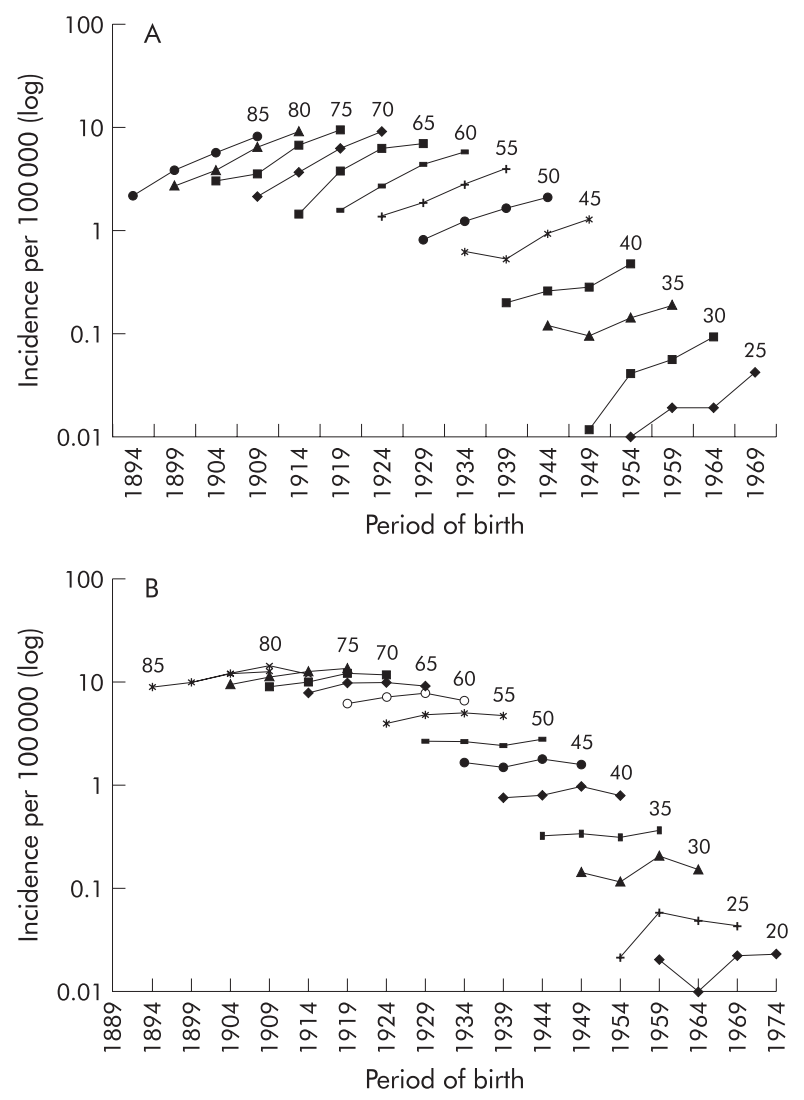

Figure 4 Birth cohort analysis of patients diagnosed with oesophageal adenocarcinoma (A) and gastric cardia adenocarcinoma (B) in the USA. The values displayed on the horizontal axis represent the middle year in a five year birth cohort-for example, 1924 for 1922-1926. Individual curves represent incidence rates in a five year age group-for example, the curve indicated as 65 is for age group 65-69. As the data represent cancer incidence during a 20 year period between 1977 and

1996, each incidence curve is comprised of four points representing the incidence rates in four separate five year birth cohorts.

years of age before declining in the oldest age groups. The age specific incidence curve during the 1992-1996 time period shifted significantly upwards, indicating a significant increase in the incidence of OA among younger subjects in addition to old age groups. Most of the $95 \%$ CIs of the incidence rates that constituted the two curves between ages 40 and 70 were nonoverlapping.

The age distribution of cases with GCA over five year periods between 1977 and 1996 is shown in fig 3B. Unlike OA, there was no significant increase in GCA age specific incidence rates among younger subjects between 1987-1991 and 1992-1996. Compared with earlier time periods, the majority of the increase in GCA cases following 1987 affected patients older than 65 years.

Figure 4A illustrates the birth cohort analysis for all patients diagnosed with OA between 1977 and 1996. Each individual curve represents age specific incidence rates in a five year age group-for example, 85 for ages 85 and older, 80 for ages $80-84$, etc. A single age specific incidence curve is composed of four points, each representing the incidence for patients from the four birth cohorts who were at the same age at the time of diagnosis. For example, the incidence curve for age 55 years is composed of incidence rates for cohorts born in 1924, 1929, 1934, and 1939, all of whom were between the ages of 55 and 59 years when diagnosed. In general, incidence rates were higher among older persons. However, irrespective of age, there was a general trend towards higher incidence rates in cohorts born more recently. This is evidenced by the 
Table 1 Odds of acquiring oesophageal adenocarcinoma and gastric cardia adenocarcinoma by birth cohort and age. Results of logiatic regression analysis

\begin{tabular}{llllll}
\hline \multirow{2}{*}{ Variable } & \multicolumn{2}{l}{ Oesophageal adenocarcinoma } & & \multicolumn{2}{l}{ Cardia adenocarcinoma } \\
\cline { 2 - 3 } \cline { 5 - 6 } & Odds ratio $(95 \% \mathrm{Cl})$ & $\mathrm{p}$ Value & & Odds ratio $(95 \% \mathrm{Cl})$ & $\mathrm{p}$ Value \\
\hline Cohort & $1.376(1.227,1.543)$ & 0.0001 & $1.000(0.925,1.080)$ & 0.9906 \\
Age & $1.066(1.060,1.072)$ & 0.0001 & $1.068(1.065,1.072)$ & 0.0001 \\
Agexcohort interaction & $1.001(0.999,1.003)$ & 0.2867 & $1.001(1.000,1.002)$ & 0.2640 \\
\hline
\end{tabular}

upward incline of all age specific incidence curves. For example, cohorts born in 1924 had a higher incidence of OA at ages 60,65 , and 70 years than those of the same ages who were born in 1919. Similarly, cohorts born in 1944 had higher incidence rates at ages 55 and 50 than those born in 1939 .

Table 1 shows the results of logistic regression analysis for OA. There was a significant cohort effect as well as a significant increase in the incidence of OA with age. The odds of acquiring OA increased by $37.6 \%$ for each five year increase in date of birth and $6.6 \%$ for each five year increase in age. No significant interactions existed between age and cohort. Conversely, the birth cohort analysis of GCA did not show a birth cohort effect. Within any same five year age group, there was no significant change in incidence rates among the different birth cohorts (fig 4B). This is evident by the flatness (equally horizontal) of the age specific incidence curves. For example, similar GCA incidence rates were observed at age 55 irrespective of whether cohorts were born in 1924, 1929, 1934, or 1939. Only age was significantly related to the incidence of GCA while the cohort effect was not significant $(p=0.99)$.

\section{DISCUSSION}

Our results indicate the presence of significant epidemiological differences between adenocarcinoma of the oesophagus and that of the gastric cardia. While the incidence of OA continues to rise in the USA, the incidence of adenocarcinoma of the cardia remained stable between 1987 and 1996. Compared with Black men, White men have approximately five times the incidence of OA but only twice the incidence of adenocarcinoma of the cardia. Concomitant with its rising incidence, OA has also significantly increased among relatively younger ages. In all age groups, the more recently born cohorts have seen a progressive rise in the incidence of OA. Such a birth cohort effect is not seen in patients with adenocarcinoma of the cardia which has remained predominantly an affliction of patients older than 65 years.

The distinction between the two diseases can be difficult, particularly in cases where the tumour involves the gastrooesophageal junction. The way these two malignancies are coded in the 9th revision of the Clinical Modification of the International Classification of Diseases (ICD-9-CM), the most commonly used disease classification in the USA, has added to the difficulty of studying them separately. In the ICD-9 CM, both malignancies are grouped together under the same code (150.0).$^{18}$ Unlike the ICD-9 CM, adenocarcinoma of the cardia and the oesophagus in the current study are distinctly classified into separate ICD-O codes that are based on specific morphological as well as histological features. ${ }^{15}$ This data source has distinct advantages over mortality or hospitalisation data contained in administrative databases where distinguishing between cancer types is not the primary purpose of the database. Nevertheless, tumour classification remains the major concern in interpreting the present results. It is often difficult, and occasionally impossible, to make a clear distinction between these tumours; such a problem cannot be solved by the available databases.

A shift in the classification as well as misclassification of gastro-oesophageal malignancies may have resulted in tempo- ral changes in their incidence rates that are not representative of changes in the true occurrence of these cancers. For example, improved diagnostic accuracy using endoscopy and autopsy may have led to diagnosing more cases of OA that would have been labelled in the past as adenocarcinoma of the cardia. However, the rates of GCA between 1987 and 1996 have levelled off while rates of OA have increased, making reclassification an unlikely cause of the observed trends in OA. Furthermore, a reclassification bias typically results in a rapid increase followed by a plateau in the incidence rates once the reclassification is widely adopted. On the other hand, among White men with gastric cancer in the USA, the proportion of cancers of unspecified site decreased from $38 \%$ in the 1970 s to $29 \%$ in the 1980s. ${ }^{19}$ Misclassification of gastric cancer as cardia cancer might explain at least some of the results of the study. Improved diagnostic accuracy may have resulted in reclassifying some cases of "unspecific site" gastric adenocarcinoma (ICD-9 CM: 151.9) ${ }^{18}$ to adenocarcinoma of the cardia ${ }^{20}$ thus contributing to the latter cancer's early rise between 1973 and 1986 that was subsequently followed by a plateau.

The US population is aging progressively and therefore the rising rates of OA could potentially reflect an "age effect" rather than a true increase in incidence. In the current results, at least three explanations make this possibility unlikely. Firstly, the use of age adjusted incidence rates in this study mitigates this effect by adjusting for the varying age structure of the population. Secondly, an increase in incidence in younger ages has accompanied the rise in OA. Finally, the birth cohort analysis has shown clearly an increase in incidence among cohorts born more recently, irrespective of their age groups. This possible cohort effect indicates the acquisition of a risk factor early in life that increases the risk of $\mathrm{OA}$ in all ages of the exposed cohort. ${ }^{16}{ }^{17}$

A few previous studies distinguished between these malignancies and found some differences in their epidemiology and risk factors. Similar to our current study, Hansen et al reported discrepancy between the temporal trends of OA and GCA incidence rates in the cancer registry of Norway. ${ }^{21}$ Between 1982 and 1992, the age adjusted rates of OA in Norway had an average annual increase of $17 \%$ in men and $15 \%$ in women, while proximal gastric cancer rates, including GCA, remained stable. Another large Swedish population based case control study was performed on the basis of the hypothesis that these tumours were distinct entities. The investigators used clear morphological and histological criteria to separate 189 cases with OA from 167 cases with GCA; both groups were then compared with 820 normal control subjects. ${ }^{10}{ }^{11}$ Among those with longstanding GORD and severe symptoms, the adjusted odds ratios were 43.5 (95\% CI 18.3-103.5) for OA and 4.4 (1.7-11.0) for GCA compared with normal controls. In the same Swedish population, a strong dose dependent relation was found between body mass index (BMI) and OA. ${ }^{11}$ The adjusted odds ratio was $7.6(3.8-15.2)$ among patients in the highest BMI quartile compared with those in the lowest. On the other hand, the odds ratio for GCA was 2.3 (1.5-3.6) in patients with the highest BMI quartile compared with those with the lowest. ${ }^{11}$ Lastly, conflicting results on the role of tobacco and alcohol were seen between studies that examined 
oesophagocardiac cancers $^{22-24}$ versus those examining each malignancy separately. ${ }^{25}$

These differences are further supported by recent studies that have indicated that risk factors for the precursor lesion of OA, Barrett's oesophagus, are different from those for IM of the gastric cardia. ${ }^{6-8}$ While GORD is a strong risk factor for Barrett's oesophagus, several studies have failed to find such an association for IM of the gastric cardia. On the other hand, $H$ pylori infection, gastritis, and the presence of IM in the distal stomach were strong predictors of the presence of IM at the gastric cardia. ${ }^{6-8}$ The presence of $\mathrm{H}$ pylori, particularly cag $A^{+}$, is inversely related to Barrett's oesophagus and OA. ${ }^{26}{ }^{27}$ Furthermore, the presence of corpus gastritis related to $H$ pylori reduces to a significant degree the risk of erosive oesophagitis. ${ }^{28-30}$ This might be related to reduced gastric acid secretion which renders the refluxate material less injurious among GORD patients. ${ }^{5}$ The relationship between $H$ pylori and adenocarcinoma of the cardia is less clear. A large US case control study reported an inverse relationship between $c a g A^{+}$ $H$ pylori and OA and GCA combined in a single group. ${ }^{30}$ In another study, Hansson et al reported a $42 \%$ seroprevalence rate of $H$ pylori in patients with "gastric cardia" tumours $(n=42)$ which was significantly lower than the $85 \%$ prevalence in gastric tumours distal to the gastric cardia. However, tumours of "gastric cardia" in the latter study were gastro-oesophageal junction tumours and hence included an unspecified number of patients with $\mathrm{OA}^{.3}$

These studies indicate that although there are obvious similarities between theses tumours, both occurring more frequently in men and Caucasians and both sharing some risk factors (GORD and obesity), there are significant differences in the magnitude of these risks as well as epidemiological variables.

It is becoming clear that when an attempt is made to separate patients with IM or adenocarcinoma at the gastrooesophageal junction into either oesophageal or cardiac, two distinctly different groups of patients are seen. Patients with Barrett's oesophagus and/or OA are mainly White, have more GORD, less $H$ pylori, less gastritis, and are increasing in number, especially among younger ages. On the other hand, patients with IM and/or adenocarcinoma of the gastric cardia are less likely to be White, tend to be older, have less GORD, more $H$ pylori, more gastritis, and are not increasing in number.

In summary, we found that the rates of OA continue to rise significantly while the rise in adenocarcinoma of the gastric cardia reported earlier has ceased after 1987. Significant differences exist between OA and GCA in sex, ethnic, age, and birth cohort distribution. These differences add further evidence in support of the hypothesis that these two malignancies are two distinct disease entities.

\section{Authors' affiliations}

H B El-Serag, Sections of Gastroenterology and Health Services Research, Houston Veterans Affairs Medical Center and Baylor College of Medicine, Houston, Texas, USA

A C Mason, C Key, University of New Mexico, Albuquerque, New Mexico, USA

N Petersen, Health Services Research Section, Houston Veterans Affairs Medical Center and Baylor College of Medicine, Houston, Texas, USA

\section{REFERENCES}

1 Blot WJ, Devesa SS, Kneller RW, et al. Rising incidence of adenocarcinoma of the esophagus and gastric cardia. JAMA 1991;265:1287-9.
2 Devesa SS, Blot WJ, Fraumeni JF Jr. Changing patterns in the incidence of esophageal and gastric carcinoma in the United States. Cancer 1998;83:2049-53

3 El-Serag HB, Sonnenberg A. Opposing time trends between esophagitis and peptic ulcer disease. Gut 1998:43:327-33.

4 Spechler SJ, Goyal RK. Barrett's esophagus. N Engl J Med 1986;315: 362-71.

5 Graham DY, Yamakoa Y. H. pylori and CagA: relationships with gastric cancer, duodenal ulcer, and reflux esophagitis and its complications. Helicobacter 1998;3:145-51.

6 El-Serag HB, Sonnenberg A, Jamal MM, et al. Characteristics of intestinal metaplasia in the gastric cardia. Am J Gastroenterol 1999;94:622-7

7 Morales TG, Bhattacharyya A, Johnson C, et al. Is Barrett's esophagus associated with intestinal metaplasia of the gastric cardia? Am J Gastroenterol 1997:92:1818-22.

8 Morales TG, Sampliner RE, Bhattacharyya A. Intestinal metaplasia of the gastric cardia. Am J Gastroenterol 1997;92:414-18.

9 Spechler SJ. The role of gastric carditis in metaplasia and neoplasia at the gastroesophageal junction. Gastroenterology 1999;1 17:2 18-28.

10 Lagergren J, Bergström R, Lindgren A, et al. Symptomatic gastroesophageal reflux disease as a risk factor for esophageal adenocarcinoma. N Engl J Med 1999;340:825-31.

11 Lagergren J, Bergström R, Nyrén O. Association between body mass and adenocarcinoma of the esophagus and gastric cardia. Ann Intern Med 1999;130:883-90.

12 Chow WH, Finkle WD, Mclaughlin JK, et al. The relation of gastroesophageal reflux disease and its treatment to adenocarcinoma of the esophagus and gastric cardia. JAMA 1995;274:474-7

13 Chow WH, Blot WJ, Vaughan TL, et al. Body mass index and risk of adenocarcinoma of the esophagus and gastric cardia. J Natl Cancer Inst 1998;90:150-5.

14 Surveillance, Epidemiology, and End Results (SEER) Program. Public-Use CD-ROM (1973-1996), National cancer Institute, DCPC, Surveillance Program, Cancer Statistics Branch, released April 1999 based on August 1998 submission.

15 World Health Organization. International Classification of Diseases for Oncology. Geneva: World Health Organization, 1976.

16 Banatavla N, Mayo K, Megraud R, et al. The cohort effect and Helicobacter pyolori. J Infect Dis 1993;168:219-21.

17 Sonnenberg A. Causative factors in the etiology of peptic ulcer disease become effective before the age of 15. J Chron Dis 1987:40:193-202.

18 World Health Organization. The international classification of diseases, 9th revision. Clinical modification, 2nd edn. DHHS Publication No. (PHS) 80-1260. Washington, DC: Public Health Service, US Government Printing Office, September 1980

19 Devesa SS, Fraumeni JF Jr. The rising incidence of gastric cardia cancer. J Natl Cancer Inst 1999:91:747-9.

20 Eksröm AM, Signorello LB, Hansson LE, et al. Evaluating gastric cancer misclassifiation: a potential explanation for the rise in cardia cancer incidence. J Natl Cancer Inst 1999;91:786-90

21 Hansen S, Wiig JN, Giercksky KE, et al. Esophageal and gastric carcinoma in Norway 1958-1992: incidence time trend variability according to morphological subtypes and organ subsites. Int J Cancer 1997:71:340-4.

22 Gray JR, Coldman AJ, Mac Donald WC. Cigarette and alcohol use in patients with adenocarcinoma of the gastric cardia or lower esophagus. Cancer 1992;69:2227-31.

23 Gammon MD, Schoenberg JB, Ahsan H, et al. Tobacco, alcohol, and socioeconomic status and adenocarcinoma of the esophagus and gastric cardia. J Natl Cancer Inst 1997;89:1277-84.

24 Zhang ZF, Kurtz RC, Sun M, et al. Adenocarcinoma of the esophagus and gastric cardia: medical conditions, tobacco, alcohol, and socioeconomic factors. Cancer Epidemiol Biomarkers Prev 1996:5:761-8.

25 Lagergren J, Bergström R, Lindgren A, et al. The role of tobacco, snuff and alcohol use in the etiology of cancer of the esophagus and gastric cardia. Int J Cancer 2000:85;340-6.

26 Weston AP, Badr AS, Topalovski M et al. Prospective evaluation of the prevalence of gastric Helicobacter pylori infection in patients with GERD Barrett's esophagus, Barrett's dysplasia, and Barrett's adenocarcinoma. Am J Gastroenterol 2000;95:387-94

27 Lord RVN, Frommer DJ, Inder S, et al. Prevalence of Helicobacter pylori infection in 160 patients with Barrett's esophagus or Barrett's adenocarcinoma. Aust NZJ Surg 2000;70:26-33.

28 El-Serag HB, Sonnenberg A, Jamal MM, et al. Corpus gastritis is protective against reflux esophagitis. Gut 1999;45:181-5.

29 Vicari JJ, Peek RM, Falk GW, et al. The seroprevalence of cagA-positive Helicobacter pylori strains in the spectrum of gastroesophageal reflux disease. Gastroenterology 1998;115:50-7.

30 Chow WH, Blaser M, Blot WJ, et al. An inverse relationship between $\operatorname{cagA} A^{+}$strains of Helicobacter pylori infection and the risk of esophageal and gastric cardia combined. Cancer Res 1998;58:588-90.

31 Hansson LE, Engstrand L, Nyrén O, et al. Prevalence of Helicobacter pylori in subtypes of gastric cancer. Gastroenterology 1995;109:885-8. 